\title{
Mean-field and mean-ensemble frequencies of a system of coupled oscillators
}

\author{
Spase Petkoski, ${ }^{1}$ Dmytro Iatsenko, ${ }^{1}$ Lasko Basnarkov, ${ }^{2,3}$ and Aneta Stefanovska ${ }^{1, *}$ \\ ${ }^{1}$ Department of Physics, Lancaster University, Lancaster LAI 4YB, United Kingdom \\ ${ }^{2}$ Ss. Cyril and Methodius University, Faculty of Computer Science and Engineering, P.O. Box 393, Skopje, Macedonia \\ ${ }^{3}$ Macedonian Academy of Sciences and Arts, Skopje, Macedonia
}

(Received 18 August 2012; revised manuscript received 21 November 2012; published 18 March 2013)

\begin{abstract}
We investigate interacting phase oscillators whose mean field is at a different frequency from the mean or mode of their natural frequencies. The associated asymmetries lead to a macroscopic traveling wave. We show that the mean-ensemble frequency of such systems differs from their entrainment frequency. In some scenarios these frequencies take values that, counterintuitively, lie beyond the limits of the natural frequencies. The results indicate that a clear distinction should be drawn between the two variables describing the macroscopic dynamics of cooperative systems. This has important implications for real systems where a nontrivial distribution of parameters is common.
\end{abstract}

DOI: 10.1103/PhysRevE.87.032908

PACS number(s): 05.45.Xt, 89.75.-k

\section{INTRODUCTION}

Systems consisting of large numbers of interacting units are common in science and nature, and have been the essential modeling tools in physics, biology, chemistry, and social science [1]. Here, the state of the whole system is characterized with macroscopic variables such as the temperature, magnetization, and so on. The values of these depend on both the microscopic laws governing the dynamics of the units as well as their interaction. Generally, only macroscopic variables are accessible in experiments. Thus their precise definition and interpretation are needed.

In the case of populations of weakly interacting oscillators, application of the phase approximation leads to the Kuramoto model (KM) for globally coupled phase oscillators [2]. Although the model itself represents an idealized scenario, its analytical tractability makes it the prevailing approach in tackling a wide variety of important problems-from Josephson-junction arrays [3] to brain dynamics under anaesthesia [4] and pedestrian induced oscillations in the Millenium bridge problem [5]. This has led to many extensions of the basic model to allow more realistic descriptions of actual systems, e.g., the KM under the influence of external fields [6], or with time-varying parameters [7] (for a review of generalizations and the problems they address see [8] and references therein).

A fundamental feature of this model is that, for a large enough coupling, synchronized behavior emerges. Depending on their inherent frequencies, some of the oscillators become locked, while the others continue to rotate asynchronously but with adjusted frequencies. The degree of the synchronization is usually characterized by some order parameter. For example, in the paradigmatic example of flashing fireflies [1], this parameter would describe the fraction of fireflies that flash in synchrony.

Since the building units of the KM are defined by their natural frequencies, the macroscopic dynamics of the oscillating system must be also characterized by some average frequency. However, two quantities can be used: the effective frequency to which synchronized oscillators are locked and the

\footnotetext{
*aneta@lancaster.ac.uk
}

average frequency of all the oscillators, locked and unlocked, that belong to the observed system. The former represents the natural macroscopic frequency, whilst the latter is the microscopically averaged mean frequency. We shall call these respectively the mean-field frequency and the mean-ensemble frequency. Returning to the example of fireflies, the frequency of those flashing in synchrony (the mean-field frequency) can generally deviate from the observed mean frequency of the whole population (the mean-ensemble frequency). Similarly, some of the neurons in the brain are expected to be mutually locked to a certain frequency, whereas an electroencephalographic recording contains the mean of all neurons in some area, not just the synchronized.

In general, there is no reason for these frequency definitions to coincide. Still, not enough attention has been paid in formulating them for different parameters. Namely, due to the equality of the frequencies in the cases with symmetry that were mostly studied, they were used interchangeably and without verification, even when they do differ (e.g., see [9]). However, here we consider scenarios which most closely resemble the actual physical or natural phenomena; in particular, models with asymmetrically distributed frequencies, phase shifted coupling function, or asymmetric couplings of opposite sign. For them we show that these frequencies always differ and have nontrivial values. Hence, one should be extremely cautious when the measured frequency of a population is interpreted and then compared with the theoretical model.

We begin with a formulation of the model and its group dynamics parameters. Section III describes the stationary solutions of the KM, whilst all possible scenarios with nontrivial mean-field and mean-ensemble frequencies are described in Sec. IV. The summary of the work and its implications are discussed in Sec. V.

\section{FORMULATION}

The KM consists of phase oscillators running at arbitrary intrinsic frequencies and coupled through the sine of their phase differences. In the case of heterogenous coupling strengths, the dynamics of the phase $\tilde{\theta}_{i}$ of the $i$ th oscillator 
has the form

$$
\dot{\tilde{\theta}}_{i}=\tilde{\omega}_{i}+\frac{K_{i}}{N} \sum_{j=1}^{N} \sin \left(\tilde{\theta}_{j}-\tilde{\theta}_{i}\right), \quad i=1, \ldots, N .
$$

Here, $K_{i}$ is the coupling strength of each oscillator and it is drawn from a probability distribution $\Gamma(K)$. Similarly, the natural frequencies $\tilde{\omega}_{i}$ are randomly distributed according to some $\tilde{g}(\tilde{\omega})$. The tildes for the frequencies, phases, and their distributions later are intentionally used for reasons to be explained below. Without loss of generality, we assume $\tilde{g}(\tilde{\omega})$ to have a mean $\left\langle\tilde{\omega}_{i}\right\rangle$ centered at 0 . Hereafter this frame of reference will be called natural. Kuramoto introduced a complex order parameter for this model, defined as a centroid of the complex representation of the oscillators

$$
\tilde{z}(t) \equiv r(t) e^{i \tilde{\psi}(t)}=\frac{1}{N} \sum_{j=1}^{N} e^{i \tilde{\theta}_{j}} .
$$

It characterizes the macroscopic behavior of the oscillators, with $r$ and $\tilde{\psi}$ being the amplitude and the phase of the mean field respectively. The former shows the level of synchronization, while the latter gives the position of the peak in the distribution of phases. Generally, the long-term values for both can depend on time. Applying Eq. (2) to the governing equation (1), it is rewritten as

$$
\dot{\tilde{\theta}}_{i}=\tilde{\omega}_{i}-K_{i} r \sin \left(\tilde{\theta}_{i}-\tilde{\psi}\right) .
$$

For infinitely large populations $N \rightarrow \infty$, a probability distribution function (PDF) $\tilde{f}(\tilde{\theta}, \tilde{\omega}, K, t)$ is defined, such that $\int_{-\pi}^{+\pi} \tilde{f}(\tilde{\theta}, \tilde{\omega}, K, t) d \tilde{\theta}=g(\tilde{\omega}) \Gamma(K)$. Thus, the complex mean field Eq. (2) becomes

$$
\tilde{z}=\int_{-\pi}^{\pi} \int_{-\infty}^{+\infty} \int_{-\infty}^{+\infty} e^{i \tilde{\theta}} \tilde{f}(\tilde{\theta}, \tilde{\omega}, K, t) d \tilde{\theta} d \tilde{\omega} d K .
$$

For convenience, infinite limits in all further definite integrals will be omitted.

As a consequence of the conservation of the number of oscillators the evolution of the density function is governed by a continuity equation

$$
\frac{\partial \tilde{f}}{\partial t}=-\frac{\partial}{\partial \theta}\left\{\left[\tilde{\omega}+\frac{K}{2 i}\left(\tilde{z} e^{-i \tilde{\theta}}-\tilde{z}^{*} e^{i \tilde{\theta}}\right)\right] \tilde{f}\right\} .
$$

Here the right-hand side of Eq. (3) is used and the sine function is expressed with complex exponents. Moreover, since $\tilde{f}$ is $2 \pi$ periodic in $\tilde{\theta}$ it allows a Fourier expansion and can be written as

$$
\tilde{f}=\frac{\tilde{g}(\tilde{\omega}) \Gamma(K)}{2 \pi}\left\{1+\sum_{k=1}^{\infty}\left[\tilde{f}_{k}(\tilde{\omega}, K, t) e^{i k \tilde{\theta}}+\text { c.c. }\right]\right\},
$$

where c.c. are the complex conjugates and $\tilde{f}_{-k}=\tilde{f}_{k}^{*}$.

In the limit $t \rightarrow \infty$, the ensemble described by (5) might settle into a stationary state for some rotating frame. We define (i) systems that have stationary solutions in some frame of reference, i.e., the complex mean field and the distribution of phases rotate uniformly and they have a constant mean field after the initial transitions; and (ii) systems that experience complex nonstable behavior, i.e., a time-varying amplitude of the order parameter $r(t)$. These definitions might differ from usual descriptions found elsewhere which regard as stationary only those solutions that are fixed in the natural reference frame. In this work, our attention is focused on the ensembles with stationary solutions as described by (i).

For the case of identically coupled oscillators with unimodal and symmetric distributions of their natural frequencies, above the critical coupling a phase locking of the oscillators takes place around the peak of $\tilde{g}(\tilde{\omega})$ where the density of the oscillators is highest [2]. As a consequence of the symmetry, the group dynamics is stationary and both mean frequencies are equal to the mode of $\tilde{g}(\tilde{\omega})$, which in this case is also its mean value.

Nevertheless, introducing multimodal or asymmetric $\tilde{g}(\tilde{\omega})$, or distributed $K$, leads to much richer dynamics. Thus, for multimodal $\tilde{g}(\tilde{\omega})$ bistabilities and standing waves emerge [10-12]. A standing wave is a macroscopic solution where neither $\tilde{f}(\tilde{\theta}, \tilde{\omega}, K, t)$ nor $\tilde{z}(t)$ are stationary in any rotating frame. This further implies nonstationarity of $r(t)$. Standing waves are also observed in systems with symmetrical bimodal distribution of natural frequencies, with the exact result for bifurcations between different states given in [13].

Traveling waves (TW), another peculiar group behavior, have also been observed for different parameter ranges within the same systems $[10,11]$. In our analysis, a TW state is considered to be any solution characterized by long-term stationarity of the mean-field amplitude, whereas the frequency of the locking $\Omega$ differs from the mean of the natural frequencies. In other words, the locking of synchronized oscillators is in a frame different from the natural. This also represents a stationary solution according to its definition above.

A recent study [9] shows the occurrence of TW in models with positive and negative coupling strengths, and identifies socalled conformists and contrarians. Similarly, a synchronization around a frequency that is different from the mean or the peak of the distribution was reported for ensembles that have an asymmetric unimodal distribution of natural frequencies [14]. It is also worth mentioning here the Kuramoto-Sakaguchi model [15], where the phase shift is introduced into the coupling function, to allow synchronization at a frequency different from the mean of the natural frequencies. Hence, it always leads to TW states. Additionally, the whole class of models of nonisochronous oscillators with constant shear can be reduced to this model $[2,16]$.

Stationary solutions for fully symmetric populations have macroscopic frequencies that are equal to $\left\langle\tilde{\omega}_{i}\right\rangle$. In asymmetric scenarios on the other hand, the synchronized cluster experiences nontrivial phase velocity $[9,14,15]$. Therefore, the focus of this work is interacting phase oscillators whose coherent behavior is characterized by a TW state, as defined earlier. Having certain asymmetries, either in the frequencies, the coupling parameters, or in the coupling function itself, is a necessary condition for occurrence of this state. As a consequence, the influence of the unsynchronized oscillators on the entrainment frequency does not vanish $[9,14,15]$. Additionally, we show that these oscillators also cause the mean-ensemble frequency to have a nontrivial value. It generally differs from the entrainment frequency, but also from the mean and the mode of the natural frequencies.

Before proceeding with the analysis of all cases with TW state, let us define the macroscopic frequencies that we have 
already discussed. The mean-field frequency represents the velocity of the mean phase $\tilde{\psi}$ and is obtained from the time derivative of the complex mean field (2),

$$
\dot{\tilde{z}} e^{-i \tilde{\psi}}=\dot{r}+i \dot{\tilde{\psi}} r=\frac{1}{N} \sum_{j=1}^{N} i \dot{\tilde{\theta}}_{j} e^{i\left(\tilde{\theta}_{j}-\tilde{\psi}\right)} .
$$

Taking into account that $r$ and $\psi$ are both real, the same is true for their time derivatives, so from Eqs. (3) and (7) one finally obtains following evolutions of the amplitude and the phase of the complex mean field:

$$
\dot{\tilde{\psi}} \equiv \Omega=\frac{1}{r N} \sum_{j=1}^{N}\left[\tilde{\omega}_{j}-K_{j} r \sin \left(\tilde{\theta}_{j}-\tilde{\psi}\right)\right] \cos \left(\tilde{\theta}_{j}-\tilde{\psi}\right) .
$$

The expression for $\dot{\tilde{\psi}}$ represents the velocity of the mean phase, i.e., the frequency of the synchronized oscillators. For the other frequency parameter-the mean frequency of the ensemble-used for characterizing these systems, its definition leads to

$$
\tilde{f}_{\text {ens }} \equiv \frac{1}{N} \sum_{j=1}^{N} \dot{\tilde{\theta}}_{j}=\frac{1}{N} \sum_{j=1}^{N}\left[\tilde{\omega}_{j}-K_{j} r \sin \left(\tilde{\theta}_{j}-\tilde{\psi}\right)\right] .
$$

In the infinite limit, (6) is introduced into (4). By taking the time derivative and applying the substitution (5), the evolution of the complex order parameter is obtained:

$$
\dot{\tilde{z}}=\iint\left[i \tilde{\omega} \tilde{f}_{1}^{*}+\frac{K}{2}\left(\tilde{z}-\tilde{z}^{*} \tilde{f}_{2}^{*}\right)\right] \tilde{g}(\tilde{\omega}) \Gamma(K) d \tilde{\omega} d K .
$$

Similarly, the mean frequency of the ensemble becomes

$$
\tilde{f}_{\text {ens }}=\int_{-\pi}^{\pi} \iint \dot{\tilde{\theta}} \tilde{f}(\tilde{\theta}, \tilde{\omega}, K, t) d \tilde{\theta} d \tilde{\omega} d K .
$$

\section{STATIONARY SOLUTIONS FOR THE PHASE DISTRIBUTION}

For a large class of problems, the long-term coherent dynamics of an ensemble of phase oscillators is time independent, as a consequence of the stationary distribution of the phases. This implies existence of the stationary solution of the continuity equation (5), which does not need to be in the natural reference frame. A recent work [17] discusses generalized empirical stability conditions for these systems.

From now on we consider that the ensemble has nonzero mean field, i.e., it is out of the incoherent state (a fully incoherent solution is also stationary), and that stationary solutions for the phase distribution exist in some planes of reference. This is true for the simplest possible scenario: unimodal symmetric frequency distribution and constant coupling strengths. The TW state is another possible scenario with this property, despite the fact that in this situation the velocity of the mean phase (or the mean-field frequency as defined here) differs from the mean of the natural frequencies.

In our analysis the mean-field frequency is allowed to be nonzero despite assuming that $\langle\tilde{\omega}\rangle=0$. Still, most of the time we work in the frame where $\tilde{f}(\tilde{\theta}, \tilde{\omega}, K, t)$ is stationary: the reference frame rotating with the frequency $\Omega$. Here the phases of the oscillators are $\theta=\tilde{\theta}-\Omega t$ and the phase corresponding to the complex order parameter is $\psi=\tilde{\psi}-\Omega t$. The distribution of the natural frequencies becomes $g(\omega)=$ $\tilde{g}(\omega+\Omega)$ with mean $-\Omega$. In the same frame $\psi=0$ can be assumed after an appropriate phase shift.

For any ensemble, if the stationary solution of (5) exists, then it exhibits two types of long-term behavior, depending on the size of $|\tilde{\omega}-\Omega|=|\omega|$ relative to $|K r|$ and to the sign of $K$. The oscillators with $|\omega|<|K r|$ approach a stable fixed point defined implicitly by

$$
\theta= \begin{cases}\arcsin \frac{\omega}{|K| r}, & \text { if } K>0 \\ \pi+\arcsin \frac{\omega}{|K| r}, & \text { if } K<0 .\end{cases}
$$

These oscillators are called locked because they maintain constant phase difference, while rotating at frequency $\Omega$ in the original frame. It is also assumed that synchronized oscillators with positive couplings have phases in the interval $(-\pi / 2, \pi / 2)$, while those with negative are in $(\pi / 2,3 \pi / 2)$, since these are necessities for stable solutions of Eq. (12) [8]. In contrast, the oscillators with $\left|\omega_{i}\right|>\left|K_{i} r\right|$ rotate in a nonuniform manner. As expected, the locked oscillators correspond to the center of $g(\omega)$ and the drifting oscillators correspond to the tails.

For the synchronized oscillators the stationary distribution of the phases becomes

$$
\begin{aligned}
& f_{s}(\theta, \omega, K, t) \\
& \quad= \begin{cases}g(\omega) \Gamma(K) \delta\left[\theta-\arcsin \left(\frac{\omega}{|K| r}\right)\right], & \text { if } K>0, \\
g(\omega) \Gamma(K) \delta\left[\theta-\arcsin \left(\frac{\omega}{|K| r}\right)-\pi\right], & \text { if } K<0 .\end{cases}
\end{aligned}
$$

Oscillators with frequencies in the interval $|\omega|>r|K|$ are out of synchrony with the mean phase. Their stationary distribution is obtained from the continuity equation (5) and the normalization condition of $f(\theta, \omega, K, t)$, such that

$$
f_{a s}(\theta, \omega, K, t)=g(\omega) \Gamma(K) \frac{\sqrt{\omega^{2}-(K r)^{2}}}{2 \pi|\omega-K r \sin (\theta)|} .
$$

Hence, the real and imaginary parts of the complex mean-field definition, Eq. (4), become

$$
\begin{gathered}
r=\int_{-\pi}^{\pi} \iint \cos \theta f(\theta, \omega, K, t) d \theta d \omega d K, \\
0=\int_{-\pi}^{\pi} \iint \sin \theta f(\theta, \omega, K, t) d \theta d \omega d K .
\end{gathered}
$$

The latter is identified as the phase balance equation.

The distribution of the phases for synchronized oscillators, Eq. (13), for each $\omega$ implies $\pi$ difference between the phases of the synchronized clusters with couplings of opposite sign. Nevertheless, this holds for the distribution $f_{s}(\omega, K, \theta, t)$ only if $g(\omega)$ is symmetric. This is not the case for the TW state, where even if the oscillators were symmetrically distributed in the natural frame, the symmetry would be broken when moving to the frame rotating with $\Omega$. Thereafter, the centroids of the phases will be shifted from the $\pi$ mutual distance, a phenomenon that was reported in [9].

In the rotating reference frame the locked oscillators are frozen; i.e., by definition $\dot{\theta}=\dot{\psi}=0$. Thus only the drifting 
ones need to be considered. This can be applied in the expression for the mean frequency of the ensemble, Eq. (11), which becomes (see the Appendix)

$$
f_{\mathrm{ens}}=\iint_{|K r|}^{\infty}[g(\omega)-g(-\omega)] \Gamma(K) \sqrt{\omega^{2}-(K r)^{2}} d \omega d K .
$$

The definition (17) is not restricted to any distributions of the natural frequencies and couplings.

Going back to the rotating frame where the original $\tilde{g}(\tilde{\omega})$ has zero mean, frequency parameters of the TW state are characterized by $\Omega$ and $\tilde{f}_{\text {ens }}$, where

$$
\tilde{f}_{\text {ens }}=f_{\text {ens }}+\Omega \text {. }
$$

As a consequence of (16) $\Omega$ can equal 0 only if $g(\omega)$ is symmetric in the boundaries of the integral in (17) [14]. This means that for any zero-centered distribution of the natural frequencies $\tilde{g}(\tilde{\omega})$ which is even in the intervals $\tilde{\omega}>|r K|$ (e.g., Lorentzian, Gaussian, etc.) the function inside the integral is also even if $\Omega=0$. Thus the integrals cancel each other and $\tilde{f}_{\text {ens }}=f_{\text {ens }}=\Omega=0$.

\section{THE TRAVELING WAVE STATES}

In the following we discuss the possible scenarios that lead to the TW state for which we also obtain mean frequency parameters described earlier.

All further analyses are carried out in the rotating frame of the entrainment frequency $\Omega$, such that $\dot{\psi} \equiv 0$ and natural frequencies are distributed according to $\tilde{g}(\omega+\Omega)$, where $\tilde{g}(\tilde{\omega})$ has zero mean. However, for better clarity of the figures, frequencies in the examples with asymmetric $\tilde{g}(\tilde{\omega})$ are depicted as seen from the original distribution described in the captions.
In other words, compared to the results from the analysis, the plots are shifted by the means of the given distributions $\tilde{g}(\tilde{\omega})$.

\section{A. Traveling waves in the Kuramoto model with contrarians and conformists}

First we focus on the TW solution described in [9], which actually inspired this work. The model shows resemblance to sociophysical models of opinion formation [18] and is also a continuation of the KM with distributed positive couplings [19].

The distribution of the couplings is

$$
\Gamma(K)=(1-p) \delta\left(K-K_{1}\right)+p \delta\left(K-K_{2}\right),
$$

where $K_{1}<0$ and $K_{2}>0$, and $p$ denotes the probability that a randomly chosen oscillator is a conformist, while $q=1-p$ is the probability that a random oscillator is a contrarian. The natural frequencies follow a zero-centered Lorentzian distribution with half-width $\gamma$,

$$
\tilde{g}(\tilde{\omega})=\frac{\gamma}{\pi\left(\tilde{\omega}^{2}+\gamma^{2}\right)} .
$$

As stated in [9], if the absolute coupling strength is higher for conformists than for the contrarians, then for some region in the parameter space $\gamma-p$, the synchronized oscillators will experience a TW. This means that both peaks in the phase distribution uniformly rotate in same direction. The waves appear in symmetric pairs, with frequencies $\pm \Omega$, since they result from the asymmetry in the coupling strengths. In contrast, if the TW is due to the asymmetry in the natural frequencies or in the coupling function, the waves are not paired, as discussed later.

Following the definitions of $\Gamma(K)$ and $\tilde{g}(\tilde{\omega})$, and using the substitution $\omega^{2}-\left(K_{1 / 2} r\right)^{2}=u_{1,2}^{2}$, the integrals in Eq. (17) are analytically solved, yielding

$$
\begin{aligned}
f_{\mathrm{ens}}=-\sqrt{2} \Omega \gamma\left\{\frac{1-p}{\sqrt{\sqrt{\left(\gamma^{2}+\Omega^{2}+K_{1}^{2} r^{2}\right)^{2}-4 K_{1}^{2} r^{2} \Omega^{2}}-\Omega^{2}+K_{1}^{2} r^{2}+\gamma^{2}}}\right. \\
\left.+\frac{p}{\sqrt{\sqrt{\left(\gamma^{2}+\Omega^{2}+K_{2}^{2} r^{2}\right)^{2}-4 K_{2}^{2} r^{2} \Omega^{2}}-\Omega^{2}+K_{2}^{2} r^{2}+\gamma^{2}}}\right\} .
\end{aligned}
$$

This expression can be straightforwardly generalized for multimodal- $\delta$ distributed coupling strengths.

It is obvious that $\Omega=0$ will imply $\tilde{f}_{\text {ens }}=0$. Hence only in the presence of a TW may the mean frequency of the ensemble differ from the mean phase velocity for this model. Similarly, for the TW state $f_{\text {ens }}$ is nonzero and has opposite sign from $\Omega$. Additionally it can be shown that the expression in the curly brackets is smaller than $1 / \gamma$, so that in the natural reference frame, $\left|\tilde{f}_{\text {ens }}\right|<|\Omega|$ always holds. This is also evident from the numerical results plotted in Fig. 1 .

Let us now derive the expression for obtaining the frequency $\Omega$ of the TW, as seen in the natural reference frame. Ott and
Antonsen, in their seminal work [20], showed that macroscopic evolution of large systems of coupled oscillators can be described by an explicit definite set of nonlinear differential equations. They introduced an ansatz for the complex Fourier coefficients in Eq. (6),

$$
\tilde{f}_{k}(\tilde{\omega}, K, t)=[\tilde{\alpha}(\tilde{\omega}, K, t)]^{k}
$$

which exactly solves the governing equation (5), as long as $\tilde{\alpha}(\tilde{\omega}, K, t)$ evolves following the nonlinear equation

$$
\frac{\partial \tilde{\alpha}}{\partial t}+i \tilde{\omega} \tilde{\alpha}+\frac{K}{2}\left(\tilde{z} \tilde{\alpha}^{2}-\tilde{z}^{*}\right)=0 .
$$




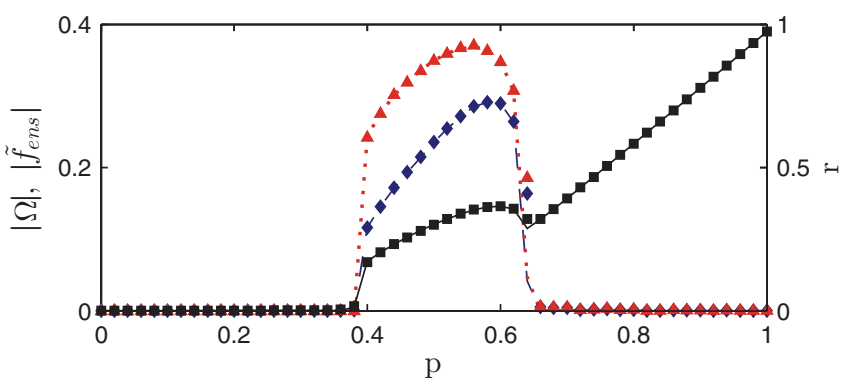

FIG. 1. (Color online) The amplitude $r$ and frequency $\Omega$ of the mean field, and the mean-ensemble frequency $\tilde{f}_{\text {ens }}$ vs the ratio $p$. Theoretical results are given with a solid line (black) for $r$, a dotted line (red) for $\Omega$, and a dashed line (blue) for $\tilde{f}_{\text {ens }}$. Results from numerical simulations are shown with squares (black) for $r$, triangles (red) for $\Omega$, and diamonds (blue) for $\tilde{f}_{\text {ens }}$. Parameters: $\gamma=0.05$, $K_{1}=-1$, and $K_{2}=2$.

When this ansatz is implemented in Eq. (4), the order parameter reduces to

$$
\tilde{z}=\iint \tilde{\alpha}^{*}(\omega, K, t) \tilde{g}(\tilde{\omega}) \Gamma(K) d \tilde{\omega} d K .
$$

Note that applying this ansatz in (10) leads to a simplified expression for the evolution of the complex order parameter,

$$
\dot{\tilde{z}}=\iint\left[i \tilde{\omega} \tilde{\alpha}^{*}+\frac{K}{2}\left(\tilde{z}-\tilde{z}^{*} \tilde{\alpha}^{* 2}\right)\right] \tilde{g}(\tilde{\omega}) \Gamma(K) d \tilde{\omega} d K,
$$

from which the frequency of entrainment can be obtained. Similarly, substituting Eqs. (3) and (6) and the ansatz (19) into Eq. (11) transforms the mean-ensemble frequency to

$$
\tilde{f}_{\mathrm{ens}}=\iint\left[\tilde{\omega}-\frac{K}{2 i}\left(\tilde{z} \alpha-\tilde{z}^{*} \tilde{\alpha}^{*}\right)\right] \tilde{g}(\tilde{\omega}) \Gamma(K) d \tilde{\omega} d K .
$$

We start by considering the low-dimensional evolution (20) in the reference frame of the TW. Similar analysis, but in the natural frame was performed in [9]. First the bimodal- $\delta$ and Lorentzian distributions for $\Gamma(K)$ and $g(\omega)$, respectively, are substituted into the integral Eq. (21), such that it yields

$$
z^{*}=(1-p) \alpha_{1}\left(\Omega-i \gamma, K_{1}\right)+p \alpha_{2}\left(\Omega-i \gamma, K_{2}\right) .
$$

This is then used in Eq. (20), which, rewritten for both $\alpha_{1}(\Omega-$ $\left.i \gamma, K_{1}\right)$ and $\alpha_{2}\left(\Omega-i \gamma, K_{2}\right)$, results in

$$
\frac{\partial \alpha_{1,2}}{\partial t}+(i \Omega+\gamma) \alpha_{1,2}+\frac{K_{1,2}}{2}\left(z \alpha_{1,2}^{2}-z^{*}\right)=0,
$$

where we omit the dependencies of $\alpha(\omega, K, t)$. As previously stated, we are interested in a stationary solution of the TW state in the $t \rightarrow \infty$ limit. This implies time-independent distribution of the phases in this limit, or from the ansatz (19), time-independent $\alpha_{1,2}$ with $\partial \alpha_{1,2} / \partial t=0$. Further, similar to [9], complex order parameters for each of the subpopulations, and the difference between their phases, are defined:

$$
r_{1} e^{-i \psi_{1}}=\alpha_{1}, \quad r_{2} e^{-i \psi_{2}}=\alpha_{2}, \quad \delta=\psi_{1}-\psi_{2}=\text { const. }
$$

In this way it is ensured that both synchronized populations, in-phase and antiphase, rotate with the same velocity $\Omega$ in the natural frame, and preserve constant phase difference. In the rotating frame of the TW $\dot{\psi}=0$, so $\psi \equiv 0$ can be set without loss of generality. Thus, from Eqs. (25) and (26) we obtain the following evolutions which describe a fixed point in the $\left\{r_{1}, r_{2}, \psi_{1}, \psi_{2}\right\}$ space:

$$
\begin{gathered}
\dot{r}_{1}=-\gamma r_{1}-\frac{K_{1}}{2}\left[\left(r_{1}^{2}-1\right)\left(p r_{2} \cos \delta+q r_{1}\right)\right]=0, \\
\dot{r}_{2}=-\gamma r_{2}-\frac{K_{2}}{2}\left[\left(r_{2}^{2}-1\right)\left(p r_{2}+q r_{1} \cos \delta\right)\right]=0, \\
\dot{\psi}_{1}=\Omega-\frac{K_{1}}{2 r_{1}} p r_{2} \sin \delta\left(r_{1}^{2}+1\right)=0, \\
\dot{\psi}_{2}=\Omega+\frac{K_{2}}{2 r_{2}} q r_{1} \sin \delta\left(r_{2}^{2}+1\right)=0 .
\end{gathered}
$$

The low-dimensional parameters including $\Omega$ can be now obtained self-consistently. The steady states (29) and (30) result from the constant angle difference between the peaks in the phase distribution, i.e.,

$$
\dot{\delta}=-\sin \delta\left[\frac{K_{1}}{2 r_{1}} p r_{2}\left(r_{1}^{2}+1\right)+\frac{K_{2}}{2 r_{2}} q r_{1}\left(r_{2}^{2}+1\right)\right]=0 .
$$

Hence, when $\operatorname{Im}[z]=0$ is applied to Eq. (24), one can use the expression

$$
q r_{1} \sin \psi_{1}=-p r_{1} \sin \psi_{2}
$$

and the definition (26) of $\delta$ to obtain the values of $\psi_{1}$ and $\psi_{2}$, such that the system will be fully described.

The equations (27)-(30) and all further numerical integrations are performed using a Runge-Kutta fourth-order algorithm. Once we have the low-dimensional parameters, Eq. (18) is applied to find the mean frequency of the ensemble. Finally, results are compared with the values for the order parameter $r$, the mean phase velocity of the ensemble $\Omega$, and the mean frequency $f_{\text {ens }}$, obtained from the numerical simulations of the ensemble Eq. (1), using Eqs. (2) and (9). As in all later simulated scenarios, the number of oscillators was set to $N=100000$; the time step of the integration was 0.01 . The simulations were running for $10^{5}$ time steps, with the initial $90 \%$ of each run discarded as possibly transient, while the rest were time averaged. The proportion of the conformists $p$ is changed from 0 to 1 at 100 equally spaced points, and the obtained results given in Fig. 1 fully confirm the theoretical analysis.

\section{B. Asymmetric unimodal frequency distribution}

Another case of the KM characterized with a stationary solution, where the mean frequency of locked oscillators differs from the mean of the oscillators' natural frequencies, are ensembles with asymmetric unimodal distribution of the natural frequencies, and equal couplings. The asymmetric scenario is also more natural than the symmetric, because any imperfection in the system, however small, can destroy the ideal symmetry. Nevertheless, mostly due to the analytical difficulties, this case has obtained little attention. It was first examined by Sakaguchi and Kuramoto [15] and the self-consistent condition for the mean-field frequency was obtained in [14].

It is known from [14] that the phase balance equation (16), for $g(\omega)$ asymmetric in the interval $|\omega|>r K$, implies that the oscillators always lock to a frequency that differs from 
the mean of the natural frequencies. For the mean-ensemble frequency Eq. (17) was numerically integrated for ensembles with triangular and log-normal frequency distributions and constant coupling strength. We found that it always gives $f_{\text {ens }}=-\Omega$ in the TW rotating frame. In other words, the mean-ensemble frequency equals the mean of the natural frequencies, although it differs from the mean-field frequency. The results seemed unexpected at first sight. However, after careful analysis we realized that the derivations for the equation of balance (16), as given in [14], in the frame rotating with $\Omega$ indeed leads to Eq. (17). Namely, applying the derivations in the Appendix, Eq. (16) becomes

$$
\begin{aligned}
0= & \Omega+\iint_{|K r|}^{\infty} g(\omega) \sqrt{\omega^{2}-(K r)^{2}} d \omega \\
& -\iint_{-\infty}^{-|K r|} g(\omega) \sqrt{\omega^{2}-(K r)^{2}} d \omega=\Omega+f_{\text {ens }} .
\end{aligned}
$$

Thus, one can conclude that, for the KM with asymmetric frequency distribution, the mean-ensemble frequency is always equal to the mean of the natural frequencies.

Next, a triangular distribution with limits $a$ and $b$, and peak at $c$ was explored. In the scenario shown in Fig. 2(a) the peak is distributed in the interval $[a, b]$, while in Fig. 2(b) the coupling strength is increasing from 4 to 10 for fixed $c$. Similarly, for log-normal frequencies

$$
\tilde{g}(\tilde{\omega})=\frac{1}{\tilde{\omega} \sigma \sqrt{2 \pi}} e^{-(\ln \tilde{\omega}-\mu)^{2} /\left(2 \sigma^{2}\right)}, \quad \tilde{\omega}>0,
$$

$\mu$ is fixed to 0 , while $\sigma$ is logarithmically distributed, and the results are given in Fig. 2(b). The mean ensemble frequency values also match theoretical values for the means of the given distributions, $(a+b+c) / 3$ and $e^{\mu+\sigma^{2} / 2}$ respectively.

For the triangular distribution shown in Fig. 2(a) the mode is $c$, while for 2(b) it is fixed. Similarly for the log-normal $\tilde{g}(\tilde{\omega})$ given in Fig. 2(c) the mode is 1 for $\mu=0$ and $\sigma \rightarrow 0$, otherwise it exponentially decreases to 0 as $e^{\mu-\sigma^{2}}$. Hence, the presented results show that the mean-field frequency is always between the mode and the mean of the distribution of natural frequencies, and reaches the latter only when all oscillators become synchronized. That is to say, by increasing the coupling strength for a given frequency distribution, the proportion of synchronized oscillators is also increased. Accordingly the value of $\Omega$ moves closer to the mean of the distribution, until it eventually reaches it for $r \rightarrow 1$. For unbounded $g(\omega)$ the last can only occur when $K \rightarrow \infty$, while in the case of bounded natural frequencies, for some value of $r K$ all oscillators will be entrained. This can also be deduced from Eq. (32). Namely, higher synchronization implies a smaller region for the integral on the right-hand side. At the same time we assume $g(\omega)$ to be decreasing left and right from the mode, meaning that higher $r$ leads to smaller value of $f_{\text {ens }}$ which eventually becomes 0 , implying $\Omega=\tilde{f}_{\text {ens }}$. Furthermore, the value under the square root in the same integral also decreases with increasing $r$. These are confirmed in Figs. 2(b) and 2(c), where we see that, for smaller couplings and hence for smaller mean field amplitudes, $\Omega$ is closer to the peak of the distribution and approaches $\tilde{f}_{\text {ens }}$ for larger coupling.
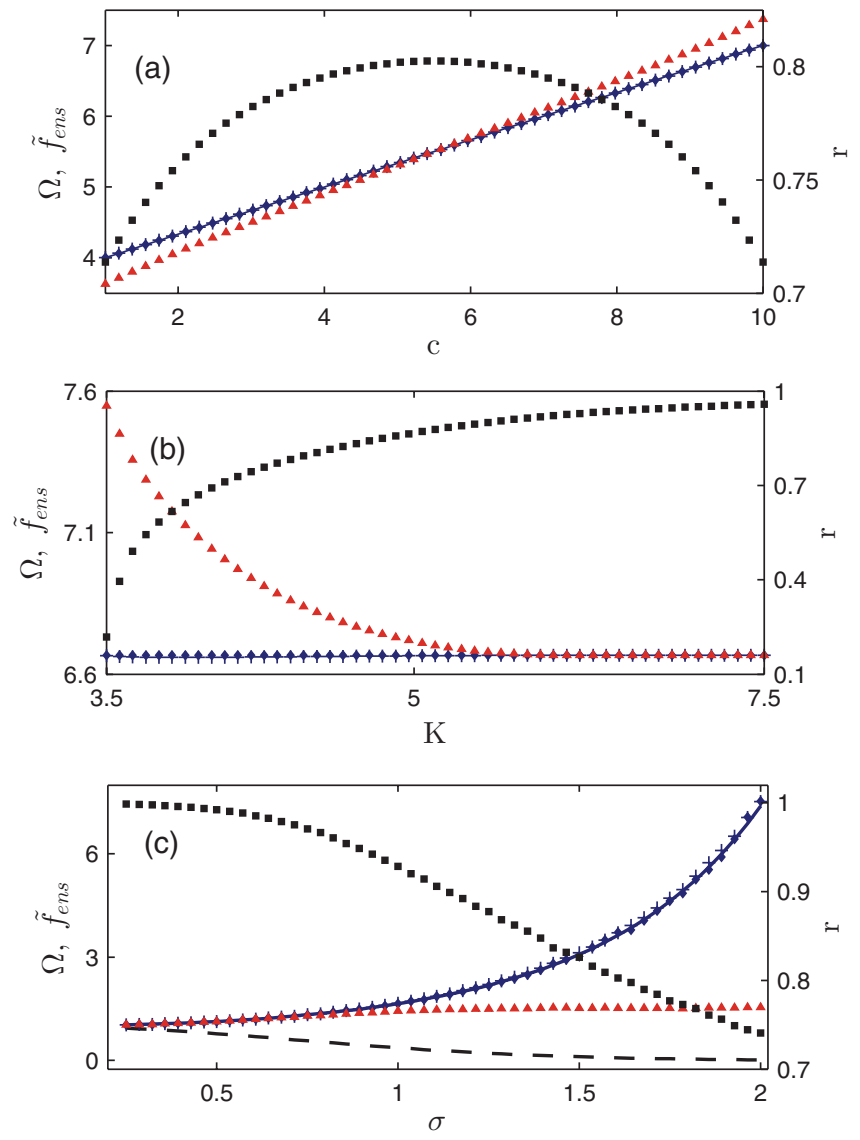

FIG. 2. (Color online) The amplitude $r$ and frequency $\Omega$ of the mean field, and the mean-ensemble frequency $\tilde{f}_{\text {ens }}$ for unimodal asymmetric natural frequencies. Results from the numerical simulations are shown with squares (black) for $r$, triangles (red) for $\Omega$, and diamonds (blue) for $\tilde{f}_{\text {ens }}$. The lines (blue) are from theoretical results for $\tilde{f}_{\text {ens }}$ and they also match the means of the frequencies' distributions: crosses (blue). (a) and (b) Triangular $\tilde{g}(\tilde{\omega})$ in boundaries $a=1$ and $b=10$. (a) Coupling $K=4.2$ and mode $c \in[0,10]$; (b) $K \in[4,10]$ and $c=9$; (c) log-normal distribution of natural frequencies, with $\mu=0, \sigma \in[0.25,2]$, and coupling $K=4.2$. The dashed line shows the modes of the distributions.

\section{Asymmetric multimodal frequency distribution}

The KM with multimodal asymmetric distribution of natural frequencies is another candidate for the mean-field behavior described by a TW. Nevertheless, due to difficulties that arise in the mathematical analysis of this model, it was never fully solved, nor has a thorough dynamical analysis of possible macroscopic solutions been performed. Still, following the analysis of the symmetric scenario [13] and qualitative descriptions of the dynamics in the asymmetric case given in [2,13], some conclusions can be drawn. Namely, Kuramoto, in his seminal work [2], discusses how transition from incoherence to mutual synchronization might be modified when the oscillators' natural frequencies are bimodally distributed. For sufficiently large coupling strength, he assumed that the clusters of synchronized oscillators "will eventually be entrained to each other to form a single giant oscillator." For smaller coupling strengths compared to the distance between peaks, he envisaged that the synchronized 
nuclei would be at the peaks of $g(\omega)$. Although some of the transitions between different states described in [2] for the symmetric case were shown to be wrong [13], the description of the mean-field dynamics of a partially synchronized state is indeed correct.

Hence, for a symmetrical $g(\omega)$ the ensemble should be either partially synchronized for large enough coupling compared to the peaks' distance, or synchronized clusters should exist near the peaks $[2,13,21]$. For asymmetrically and bimodally distributed frequencies the partial synchronization will be characterized by TW, whereas standing waves and other complex collective rhythms appear for smaller couplings.

Therefore, for the partially synchronized state in the asymmetric bimodal case, one might expect a TW state to occur. As a consequence of having a single synchronized cluster, the dynamic of the system is similar to the models with unimodal asymmetric distribution described in Sec. IV B. As a result the same conclusion drawn about the asymmetric unimodal scenario also holds for the TW solution of the asymmetric bimodal case. This means that the mean frequency of the ensemble is equal to the mean of $\tilde{g}(\tilde{\omega})$, and as such differs from the frequency of the TW experienced by the mean field. Hence, in the TW frame, $f_{\text {ens }}=-\Omega$. This can be seen in Fig. 3, where results from numerical simulations confirmed the theoretically expected values for $\tilde{f}_{\text {ens }}$ and $\Omega$.

As an example we analyze the dynamics of a population with bimodal Lorentzian distribution of frequencies

$$
\tilde{g}(\tilde{\omega})=\frac{(1-p) \gamma_{1}}{\pi\left[\left(\tilde{\omega}-\mu_{1}\right)^{2}+\gamma_{1}^{2}\right]}+\frac{p \gamma_{2}}{\pi\left[\left(\tilde{\omega}-\mu_{2}\right)^{2}+\gamma_{2}^{2}\right]} .
$$

The subdistributions are peaked at $\mu_{1}$ and $\mu_{2}$ with the halfwidths $\gamma_{1}$ and $\gamma_{2}$ respectively, where $p$ is the proportion of the oscillators belonging to the second subdistribution. The full low-dimensional dynamics for this system can be obtained using the ansatz Eq. (19) in a similar manner as in Sec. IV A. The integral (21) for the given $\Gamma(K)$ and $g(\omega)$ yields

$$
z^{*}=(1-p) \alpha_{1}\left(\mu_{1}-\gamma_{1}, K, t\right)+p \alpha_{2}\left(\mu_{1}-\gamma_{1}, K, t\right) .
$$

Hence, substituting $\alpha_{1,2}$ in Eq. (20), the low-dimensional dynamics are described by two ODEs,

$$
\dot{\alpha}_{1,2}=-\left(i \mu_{1,2}+\gamma_{1,2}\right) \alpha_{1,2}-\frac{K}{2}\left(z^{*}+z \alpha_{1,2}\right) .
$$

In all the plots in Fig. 3 the values of the coupling strength are large enough to induce TW instead of standing wave states, i.e., one instead of two synchronized clusters. For Fig. 3(a) both peaks are of equal distance from the zero. This leads to zero mean for any width of the subdistributions, and following previous discussion and Eq. (32) it implies $\tilde{f}_{\text {ens }}=0$. For similar deviations of subdistributions the entrainment frequency will be near zero, but closer to the narrower peak, since more oscillators from that subdistribution will be entrained. However, for a large deviation of the first subdistribution compared to the second one, a small number of the oscillators belonging to it will be entrained. These oscillators will be almost equally distributed on both sides of the second peak. Thus, the frequency of the entrained oscillators will asymptotically reach the peak of the second subdistribution in the limit case $\gamma_{1} \rightarrow \infty$. If in the same limit case $p \rightarrow 0$ also holds, then with the same reasoning very
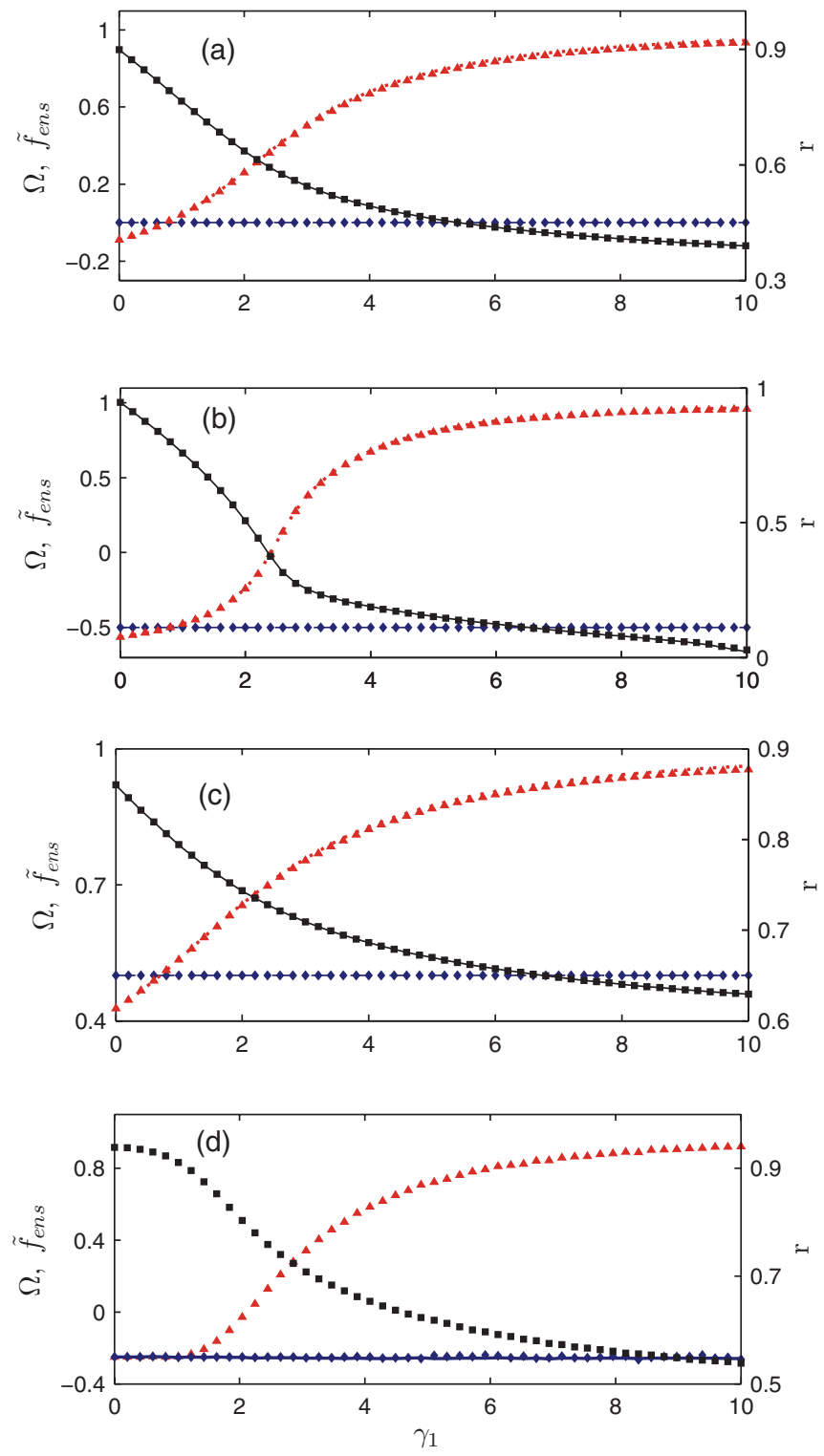

FIG. 3. (Color online) The amplitude $r$ and frequency $\Omega$ of the mean field, and the mean-ensemble frequency $\tilde{f}_{\text {ens }}$ vs the frequency width of the first subpopulation $\gamma_{1}$. Results from the simulations, Eq. (1), are shown with squares (black) for $r$, triangles (red) for $\Omega$, and diamonds (blue) for $\tilde{f}_{\text {ens }}$. (a)-(c) The low-dimensional dynamics, Eq. (33), are shown with solid lines (black) for $r$, dotted lines (red) for $\Omega$, and dashed lines (blue) for $\tilde{f}_{\text {ens. }}$. (a)(c) Bimodal Lorentzian $\tilde{g}(\tilde{\omega})$. Parameters: $\gamma_{1} \in[0,10] \gamma_{2}=0.75$, $\mu_{1}=-1, \mu_{2}=1, K=5$, and (a) $p=0.5$, (b) $p=0.25$, and (c) $p=0.75$. (d) Bimodal Gaussian $\tilde{g}(\tilde{\omega})$ with $\gamma_{1} \in[0,10], \gamma_{2}=$ $0.75, \mu_{1}=-1.5, \mu_{2}=1$, coupling $K=4.25$, and $p=0.5$.

few entrained oscillators will be close to the second peak. At the same time the mean frequency of the distribution and of the ensemble will be near the first peak. Similarly, for $p=0.25$ and $p=0.75$ as in Figs. 3(b) and 3(c), the mean of the frequencies is on $1 / 4$ or $3 / 4$ of the distance between the peaks, respectively, while $\Omega$ reaches the narrower mode.

The generality of the described analysis for any bimodal distribution is shown in Fig. 3(d), where the frequency distributions are Gaussian. Although the low-dimensional 
dynamics in a form similar to Eq. (33) cannot be obtained for this case, the results from the numerical simulations of the ensemble, Eq. (1), are in line with the previous discussion concerning Lorentizan $\tilde{g}(\tilde{\omega})$.

\section{Contrarians and conformists with asymmetric unimodal frequency distribution}

The analysis naturally continues with the KM in the presence of both previously analyzed conditions that are required for TW: contrarians and conformists, with asymmetric distribution of natural frequencies. The phase locking in this scenario happens in frames other than the natural for all coherent solutions. But as it will be shown, due to the distributed couplings, the mean ensemble frequency also has nontrivial values; it is not always equal to $\langle\tilde{\omega}\rangle$. As another consequence of the asymmetry, the sign of the TW can no longer be expected to be random, as is the case for the contrarians and conformists over symmetrically distributed frequencies [9]. However, the analysis of this case is largely more complicated compared to previous ones and we have taken some examples only to show the main points.

The phase balance equation (16) for distributed $K$ in this case does not lead to a simple expression, as was Eq. (32) for equal couplings. Still, following the same procedure, Eqs. (13) and (14) are substituted into Eq. (16) and, applying the derivations from the Appendix, the balance of phases becomes

$$
\begin{aligned}
0= & \int \frac{\Gamma(K)}{r K} d K\left\{\int \omega g(\omega) d \omega\right. \\
& \left.-\int_{|K r|}^{\infty} \omega[g(\omega)-g(-\omega)] \sqrt{\omega^{2}-(K r)^{2}} d \omega\right\} \\
= & \int \frac{\Gamma(K)}{r K} d K[-\Omega+I(K)] .
\end{aligned}
$$

This expression self-consistently gives the frequency of the entrainment, whilst it no longer implies equality of $\tilde{f}_{\text {ens }}$ and $\langle\tilde{\omega}\rangle$, as for constant coupling parameters. Results from numerical simulations shown in Fig. 4 confirm the nontrivial nature of both global frequency parameters. The most interesting phenomena is that $\Omega$ and $\tilde{f}_{\text {ens }}$ for some parameters can have values that not only differ from either the mode and the mean of the natural frequencies, but that are also outside of the interval between them as was case for equal $K$. Moreover, they can even be outside of the boundaries of their distribution. For example for the triangular $\tilde{g}(\tilde{\omega}) \in[0,1]$ depicted in Fig. 4(a), the frequency of the entrainment can be up to $\sim-1$ or to $\sim 2$, and similar results occur with $\tilde{f}_{\text {ens }}$. These values are clearly out of the region of support of $\tilde{\omega}$, which is shaded in the same plot. Note that these on first sight counterintuitive results are a consequence of the very high coupling strengths with opposite sign, and they are still within the general boundaries of $\tilde{f}_{\text {ens }}$ and $\Omega$. Namely, from Eqs. (11) and (3), it is clear that

$$
\hat{\omega}-\max |K|<\tilde{f}_{\text {ens }}<\hat{\omega}+\max |K|,
$$

while from the fact that the entrainment frequency cannot be out of the limits $[\min (\dot{\tilde{\theta}}), \max (\dot{\tilde{\theta}})]$ it follows

$$
\min (\tilde{\omega})-\max |K|<\Omega<\max (\tilde{\omega})+\max |K| .
$$
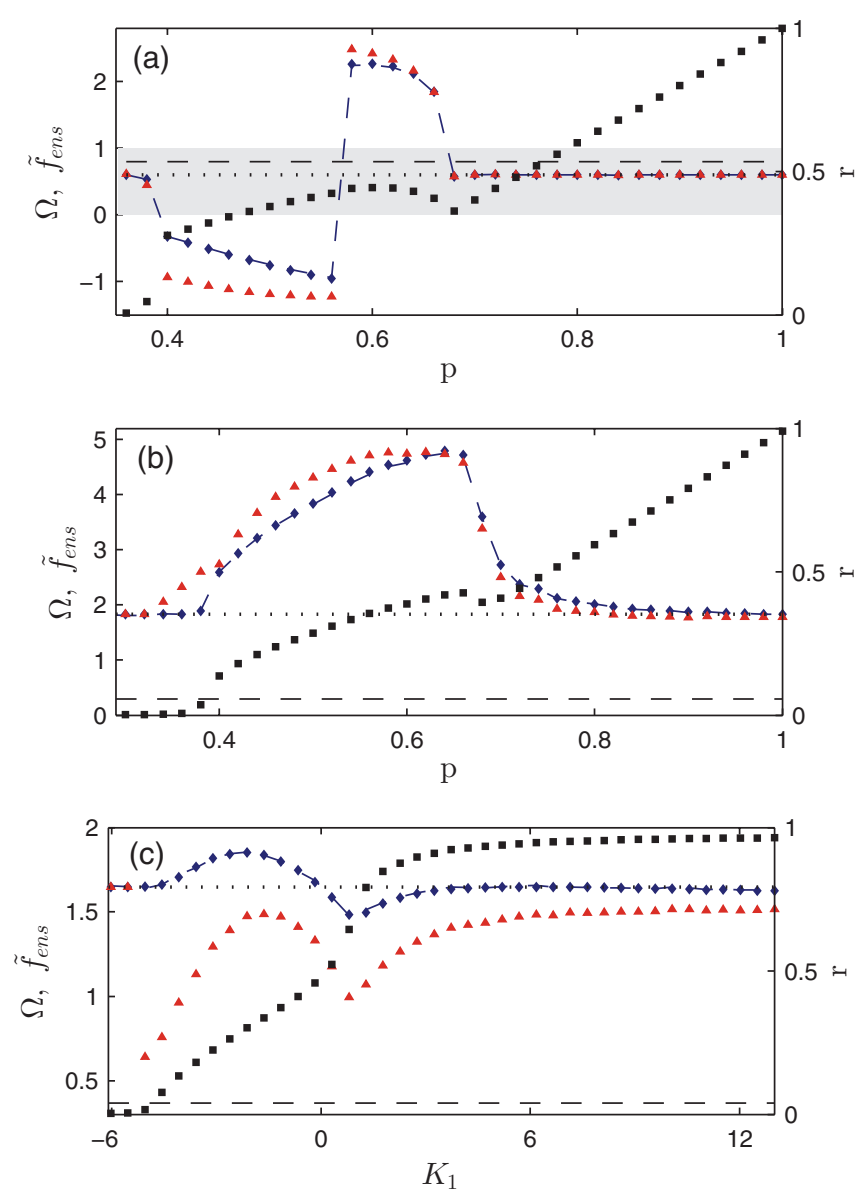

FIG. 4. (Color online) The amplitude $r$ and frequency $\Omega$ of the mean field, and the mean-ensemble frequency $\tilde{f}_{\text {ens }}$ for asymmetric ensembles with distributed coupling strengths. Numerically obtained results, Eq. (1), are shown with squares (black) for $r$, triangles (red) for $\Omega$, and diamonds (blue) for $\tilde{f}_{\text {ens }}$. (a)-(c) The dashed lines (blue) are theoretically predicted results for $\tilde{f}_{\text {ens }}$, Eq. (17). Horizontal dashed and dotted lines are the modes and the means of $\tilde{g}(\tilde{\omega})$ respectively, and its domain is shaded for (a). (a) Triangular $\tilde{g}(\tilde{\omega})$ within $a=0$ and $b=$ 1 and peak at $c=0.8$; bimodal- $\delta \Gamma(K)$ with $K_{1}=-4, K_{2}=8$, and $p \in[0.34,1]$. (b) and (c) Log-normal $\tilde{g}(\tilde{\omega})$ with $\mu=0$ and bimodal- $\delta$ $\Gamma(K)$. (b) $\sigma=1.1, K_{1}=-10, K_{2}=20$; and (c) $\sigma=1, p=0.55$, $K_{1} \in[-6,13], K_{2}=6$.

Of course, these are broad limits and only for bounded $\tilde{g}(\tilde{\omega})$ do the boundaries for $\Omega$ not reach $\pm \infty$.

Hence, for bounded distributions, such as the triangular, and for large enough couplings of opposite signs, the only way the balance equation (34) can be satisfied is with the emergence of a TW with a large enough value of $\Omega$, so that the integral in the same equation will be nonvanishing. Contrarily, if $I(K)=0$ for the shown example, i.e.,

$$
\tilde{\omega}-\Omega<|r K|, \quad \forall \tilde{\omega} \text { for which } \tilde{g}(\tilde{\omega})>0,
$$

then the phase balance becomes

$$
0=(1-p) / K_{1}+p / K_{2} \text {. }
$$

If (38) holds, then $\Omega$ can have any value that still obeys condition (37), so each simulation would give a different TW within these limits. Another consequence from condition (37) when applied to Eq. (17) is that $f_{\text {ens }}=0$. 
If (38) is not satisfied, then the oscillators rearrange, rendering the condition (37) also invalid. The result is the appearance of nonzero $I(K)$ in Eq. (34) to impose the balance of the phases. There may be more than one value for $\Omega$ which impose this balance, and if that were the case one might expect multiple solutions or even hysteresis behavior. In this work, however, we only numerically confirm that for bounded natural frequencies, as in the example in Fig. 4(a), $\Omega$, when observed in the natural frame, can have two stable values with opposite signs. In other words, numerical realizations could lead to any of the two stable values. This is illustrated in Fig. 4(a) where one such realization is depicted, whilst in different realizations the values on both sides of the mean and the mode of $\tilde{g}(\tilde{\omega})$ appeared for $p$ around $(0.35,0.7)$.

For the same triangular natural frequencies, if $p>0.65$, then a state similar to the $\pi$ state in the symmetric model in Sec. IV A is observed. Here, the equality of $\Omega$ and $\tilde{f}_{\text {ens }}$ follows from the bounded distribution of the oscillators' natural frequencies, which all become entrained. Similarly the integral $I(K)$ vanishes, and the requirement for the phase balance holds only if $\Omega=0$. In this way only the first integral of Eq. (34) survives and it gives $\langle\tilde{\omega}\rangle$, which we set to be 0 [although in Fig. 4 the plots are for nonzero mean frequency distributions, such that the plot (a) has mean 0.8].

If the natural frequencies are unbounded then $I(K) \neq 0$, meaning that a TW always emerges. For the simulations we have performed, as seen in Figs. 4(b) and 4(c), the system was always setting the same value for $\Omega$ that solves Eq. (34) for the given parameter range. Thus, for the log-normally distributed $\omega, \Omega$ is always positive, although the existence of another stable TW that also fulfills the phase balance is not excluded. The entrainment of all oscillators is never achieved in this scenario, and the mean frequencies will never reach $\langle\tilde{\omega}\rangle$. Still they will approach this value when the number of entrained oscillators is increased, either by increasing the number of conformists, Fig. 4(b), or by changing the coupling strength of one of the groups, as shown in Fig. 4(c). The same plots also show that, even when the couplings are all positive, the group frequencies behave qualitatively differently from the case with constant $K$. This characteristic becomes less obvious once the modes are closer to each other. To check the generality of the analysis, we also explored the case when $K$ is bimodal Gaussian distributed. The results did not show any qualitative difference from the case with bimodal $\delta$.

It is expected that, for multimodal couplings, clustering emerges and plays a crucial role in defining the values of $\tilde{f}_{\text {ens }}$ and $\Omega$. A similar phenomenon was also reported in [19] for distributed positive and unimodal $K$, but as a finite-size effect only. That is to say, the distribution of the locked oscillators, Eq. (13), for multimodal- $\delta \Gamma(K)$ will have peaks on different angles $\psi_{i}=\arcsin \frac{\omega}{K_{i} r}$ for each mode $K_{i}$. Further evidence for this explanation can be seen in numerical simulations performed over different $\Gamma(K)$ and $g(\omega)$. Figure 5 presents obtained phase distributions. As can be seen three qualitatively different types were identified.

Firstly for very large coupling strengths compared to the width of $g(\omega)$, and for bimodal $\Gamma(K)$ with modes having different signs, there are two separated peaks corresponding to the contrarians and conformists. The distance between them is smaller than $\pi$ and the observed TW is similar to that in
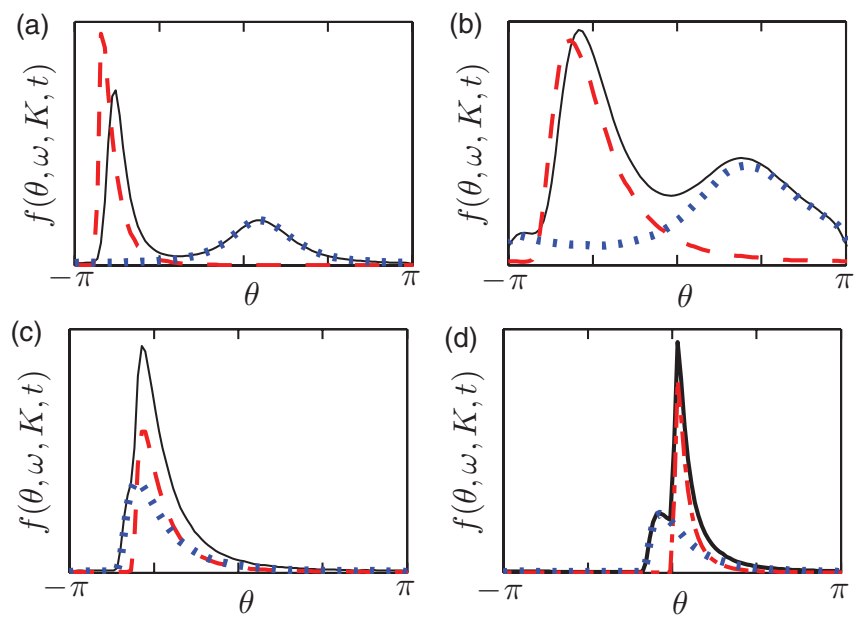

FIG. 5. (Color online) PDF of phases for different states of the ensemble with log-normally distributed natural frequencies and bimodal- $\delta$ distributed coupling strengths. Oscillators with coupling $K_{1}$ are shown with a dashed line (red), those with $K_{2}$ are following the dotted line (blue,) while the joint distribution is shown with a solid line (black). Parameters: $\mu=0, \sigma=1$, and $p=0.5$. (a) $K_{1}=-10$, $K_{2}=20$. Two peaks at a distance smaller than $\pi$ and a TW occurs. (b) $K_{1}=-4, K_{2}=8$. Two peaks at a distance $\pi$. (c) $K_{1}=2$, $K_{2}=3$. The peaks merge into a single peak in the joint distribution. (d) $K_{1}=2, K_{2}=6$. Two closely separated peaks.

Sec. IV A, although the asymmetry in $g(\omega)$ causes asymmetry in the distribution $f(\theta, \omega, K, t)$. This is shown in Fig. 5(a), and also in examples (a) and (b) of Fig. 4, in the range for $p$ around $0.4-0.65$.

The second scenario differs from the first only in the sense that the absolute values of the coupling strengths are not much bigger than the width of the natural frequencies. As expected, this corresponds to the $\pi$ state from [9], but the form of $g(\omega)$ will be mapped onto the peaks which are separated by $\pi$. The asymmetry additionally will influence the values of the $\tilde{f}_{\text {ens }}$ and $\Omega$. However, the latter will always be in the interval between the mode and the mean of $\tilde{g}(\tilde{\omega})$. This means that qualitatively this corresponds to the case with constant $K$ described in Sec. IV B, but with nonzero values for $\tilde{f}_{\text {ens }}$ because of the phase balance equation (34).

Lastly, if the couplings distribution have positive peaks only, depending on their distance apart, the phases will have either one or two close peaks, while still keeping the form of $g(\omega)$. Hence, the case with one peak will be the same as having unimodally distributed $K$, and numerical results suggest that $\tilde{f}_{\text {ens }} \approx\langle\tilde{\omega}\rangle$ as for constant $K$, although this is not immediately obvious from Eqs. (17) and (34). Finally, if $f(\theta, \omega, K, t)$ is no longer unimodal, then $\tilde{f}_{\text {ens }}$ has values that differ from $\langle\tilde{\omega}\rangle$.

The number and the nature of the emerging clusters is not so straightforward for non- $\delta \Gamma(K)$ distributions. Still, from the $\mathrm{PDF}$ of the phases it is clear that, for negative modes in $\Gamma(K)$, the peaks in the PDF corresponding to those oscillators do not have the form of $g(\omega)$, but they are more symmetric. For positive modes, the peaks keep mapping the distribution of the natural frequencies. This is also an interesting peculiarity that requires further attention. 
The bottom line is that different, and on first sight counterintuitive values for the entrainment and mean ensemble frequency are obtained even for all positive but multimodally distributed couplings, as long as the natural frequencies are nonsymmetric. Nevertheless, a thorough analysis is still needed for this general case of the KM, with the emphasis put on the entrainment at different clusters and their influence to the group behavior.

\section{E. Phase shifted coupling function}

The final model leading to TW of the macroscopic parameters being analyzed here is actually the earliest generalization of the KM [15]. This model was introduced to allow for entrainment in frames other than the natural. The so called Kuramoto-Sakaguchi model has the form

$$
\dot{\tilde{\theta}}=\tilde{\omega}-K r \sin \left(\tilde{\theta}_{i}-\tilde{\psi}-\beta\right),
$$

where $\beta$ is a phase shift of the coupling function. Lowdimensional dynamics of this model with Lorentzian $\tilde{g}(\tilde{\omega})$ can be also obtained using the ansatz [20], with derivations being similar to the problem in Sec. IV A. It yields

$$
\frac{\partial \tilde{z}}{\partial t}+\gamma \tilde{z}+\frac{K}{2}\left(\tilde{z} \tilde{\alpha}^{2} e^{i \beta}-\tilde{z}^{*} e^{-i \beta}\right)=0
$$

which was also obtained in [22]. From here, one immediately obtains the long-term evolution of $\psi$, i.e., the frequency of the TW,

$$
\frac{d \tilde{\psi}}{d t} \equiv \Omega=K \sin \beta-\gamma \tan \beta .
$$

For the mean ensemble frequency in this model, Eq. (17) can be analytically solved, and with further substitution of stationary values for $r$ and $\Omega$ it transforms to

$$
\tilde{f}_{\text {ens }}=-\operatorname{sgn}(\Omega) \gamma \tan \beta .
$$

This directly leads to

$$
\tilde{f}_{\text {ens }}=K \sin \beta-2 \gamma \tan \beta,
$$

which can also be straightforwardly obtained from Eq. (23) after applying the residue theorem for the integral over $\omega$. The stationary values of the macroscopic parameters of this system are illustrated in Fig. 6. There, it is also obvious that $\left|\tilde{f}_{\text {ens }}\right|<|\Omega|$ and that the frequencies have odd symmetry, features which are a direct consequence of Eqs. (39) and (40).

It is worth noting that by taking the derivative over $\beta$ from expressions (39) and (40), extreme values for both mean frequencies follow as

$$
\beta_{\max / \min }= \pm \arccos \sqrt[3]{2 \gamma / K}
$$

for $\Omega$ and

$$
\beta_{\max / \min }= \pm \arccos \sqrt[3]{\gamma / K}
$$

for $\tilde{f}_{\text {ens. }}$. Of course, since the population should be coherent, parameters have to be chosen such that $K>K_{c}=2 \gamma / \cos \beta$ holds. Thus, the values of the phase shift $\beta$ that produce maximum deviation in both mean-ensemble and mean-field frequencies are directly obtained.

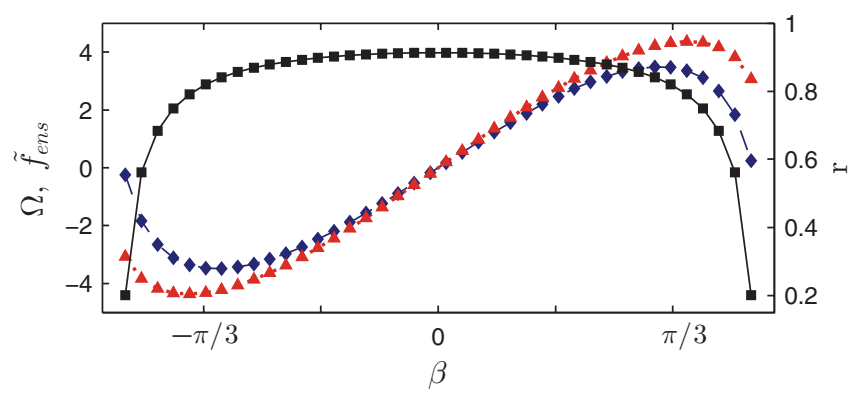

FIG. 6. (Color online) The mean field's amplitude $r$, the phase velocity $\Omega$, and the mean frequency of the ensemble $\tilde{f}_{\text {ens }}$ vs the phase shift $\beta$. Results from the theoretical predictions for the lowdimensional dynamics, Eqs. (39) and (40), are given with a solid line (black) for $r$, a dotted line (red) for $\Omega$, and a dashed line (blue) for $\tilde{f}_{\text {ens. }}$. Results from numerical simulations, Eq. (1), of the ensemble are shown with squares (black) for $r$, triangles (red) for $\Omega$, and diamonds (blue) for $\tilde{f}_{\text {ens }}$. Parameters: $\gamma=0.5, K=6$, and $\alpha \in[-4 \pi / 9,4 \pi / 9]$.

\section{SUMMARY}

The TW state in the KM occurs whenever the symmetry in either the natural frequencies or the coupling function itself is broken. For asymmetric coupling strengths on the other hand, the wave occurs only for certain coupling parameters. The results of this study indicate that, in populations with an asymmetric, bell-shaped distribution of the nonsynchronized oscillators and equal couplings, the mean-ensemble frequency is always the mean of the natural frequencies. In contrast, the TW of the synchronized oscillators-the mean-field frequency-has a value between the mean and the mode of those. When the asymmetry originates from the coupling strengths or the coupling function, both mean frequencies have nontrivial values. In particular they differ from the mean or the modes of the frequency distribution.

The case of the asymmetric unimodal frequency distribution has quite a straightforward explanation. Here the frequency of synchronization is a result of the interplay between the cluster of the locked oscillators and the whole ensemble. Synchronized oscillators tend toward frequency locking at the mode, where the density of similar oscillators is the highest, whilst the influence of all oscillators would be balanced if it is at the mean of all natural frequencies. The compromise is achieved through the self-consistent system for the phase balance, and Eq. (32) follows directly from this interplay. This balance would be destroyed if the nucleus is either on the peak or on the mean.

By increasing the number of locked oscillators, the meanfield frequency shifts towards the less skewed side of the distribution, i.e., towards the mean, because the number and the influence of the drifting oscillators decreases. Finally, once all the oscillators get locked, the frequency of the entrainment will be exactly the mean of the distribution. In this way, the influence of all oscillators is equal. The same explanation can be applied for bimodal or multimodal cases, since again there is only one cluster of synchronized oscillators in the TW state.

On the other hand, in the case of contrarians and conformists, the physical explanation of the mean-ensemble frequency, the frequency of entrainment, and their difference, is not yet very clear. Previous work went as far as showing 
that, in some parameter spaces for opposite sign couplings, both peaks in the distribution of the phases lose their stability. Instead, they start to chase each other and are no longer a distance of $\pi$ apart, hence producing the TW. Here we have emphasized that this behavior simply acts to preserve the phase balance, which directly gives the value of the entrainment frequency, while its influence on arranging the nonsynchronized oscillators also makes it responsible for the mean ensemble frequency. Despite this, we believe that, even by showing, analytically and numerically, that the velocity of the mean phase and the frequency of the ensemble differ in this case, an important characteristic that seems to be unique for TW states only is immediately revealed. This explanation was missing when the model was first introduced [9], and clear distinction between macroscopic frequencies was not made. Moreover, "the mean phase velocity" obtained through the low-dimensional dynamics was defined as the mean-ensemble frequency.

In the more general and realistic situation, with asymmetric frequencies and distributed couplings, the macroscopic frequencies can have values in very unexpected boundarieseven outside the limits of the natural frequencies. The reason for this complex behavior is again a result of the system's self-arranging, such that the influence of the nonsynchronized oscillators to the mean-field amplitude vanishes and the phase balance is maintained. In order for this to be achieved for multimodally distributed couplings, there are different clusters of synchronized oscillators for each mode. This case also shows possibilities for further research either in eventual hysteresis dynamics and seeking the number of stable solutions, or in the clustering phenomena for distributed coupling strengths.

When the asymmetry is induced through the coupling function itself the macroscopic frequencies always differ, in a similar way to the case when it stems from the coupling parameters. In that sense the mean-ensemble frequency is of the same sign, but with lower absolute value than the mean-field frequency. Also, their dependence on the phase shift is nonlinear, and the ways they respond to the shift do not coincide, with both of them having the highest values for different phase shifts.

Taken together, these results suggest that, whenever the population is experiencing a TW, the locking of the oscillators is at a frequency different from the mean of the instantaneous frequencies. Since in inverse problems or in experiments it is often only the macroscopic parameters that can be obtained, a clear interpretation of the observed mean frequency is always needed. Moreover, the asymmetric scenarios tend to be far more abundant in the real physical systems. That is to say, asymmetry of the natural frequencies can immediately make any scenario more realistic, whilst few of the examples with opposite coupling strengths can be traced in inhibitory and excitatory neurons [23] or in social dynamics [18]. As for the phase-shifted coupling function, although it was introduced to describe the formation of nonlinear waves in nonoscillatory media [15], it can be used to model various phenomena, such as Josephson junctions [3], mammalian intestines, and heart cells [24]. Hence, in the models based on measurements, like those describing the brain dynamics [4], one should precisely define to which of the macroscopic frequencies, of the mean field or mean ensemble, the measured frequency corresponds.
Finally, this work reveals the strong need for future research that should explain the physical link between the observed mean frequency of any system with cooperative dynamics and the two macroscopic frequency parameters described here for nontrivial cases.

\section{ACKNOWLEDGMENTS}

The study was supported by the EPSRC; S.P. is supported by the Lancaster University Ph.D. grant and L.B. was supported by Faculty of Computer Science and Engineering at the Ss. Cyril and Methodius University and ERC Grant No. 266722 (SUMO project). We are grateful to Gemma Lancaster and Phil Clemson for their useful comments on the manuscript.

\section{APPENDIX: GENERAL FORMULA FOR THE MEAN ENSEMBLE FREQUENCY}

We work in the reference frame rotating with $\Omega=\dot{\tilde{\psi}}$. In this frame, the locked oscillators are frozen and one has to consider the drifting ones only, Eq. (14). This can be applied in the expression for the mean frequency of the ensemble, Eq. (11), which becomes

$$
\begin{aligned}
f_{\text {ens }}= & \int_{-\pi}^{\pi} \iint_{|\omega|>|K r|}(\omega-K r \sin \theta) g(\omega) \Gamma(K) \\
& \times \frac{\sqrt{(\omega)^{2}-(K r)^{2}}}{2 \pi|\omega-K r \sin (\theta)|} d \omega d K d \theta \\
= & I_{1}-I_{2} .
\end{aligned}
$$

The first integral is

$$
I_{1}=\iint_{|\omega|>|K r|} \omega \Gamma(K) g(\omega) d K d \omega,
$$

because of the probability normalization

$$
\int_{0}^{2 \pi} \frac{\sqrt{(\omega)^{2}-(K r)^{2}}}{2 \pi|\omega-K r \sin \theta|} d \theta=1 .
$$

The second integral requires more calculation. Integrating over the phases first, for positive frequencies $\omega>|K r|$ it yields

$$
\int_{0}^{2 \pi} \frac{\sqrt{\omega^{2}-(K r)^{2}} K r \sin \theta}{2 \pi|\omega-K r \sin \theta|} d \theta=\omega-\sqrt{\omega^{2}-(K r)^{2}},
$$

while for negative frequencies $\omega<-|K r|$

$$
\int_{0}^{2 \pi} \frac{\sqrt{\omega^{2}-(K r)^{2}} K r \sin \theta}{2 \pi|\omega-K r \sin \theta|} d \theta=\omega+\sqrt{\omega^{2}-(K r)^{2}} .
$$

It is interesting to note that both integrals are even in $K$. Following this, the second integral, $I_{2}$, simply becomes

$$
\begin{aligned}
I_{2}= & \int_{-\infty}^{\infty} \int_{-\infty}^{-|K r|} g(\omega) \Gamma(K)\left[\omega+\sqrt{\omega^{2}-(K r)^{2}}\right] d \omega d K \\
& +\int_{-\infty}^{\infty} \int_{|K r|}^{\infty} g(\omega) \Gamma(K)\left[\omega-\sqrt{\omega^{2}-(K r)^{2}}\right] d \omega d K .
\end{aligned}
$$

After partial cancellation of $I_{2}$ with $I_{1}$ because of (A1) one obtains a simple formula for calculation of the mean ensemble 
frequency in the reference frame of the order parameter:

$$
\begin{aligned}
f_{\mathrm{ens}}= & \int_{|K r|}^{\infty} \int_{-\infty}^{\infty} g(\omega) \Gamma(K) \sqrt{\omega^{2}-(K r)^{2}} d \omega d K \\
& -\int_{-\infty}^{-|K r|} \int_{-\infty}^{\infty} g(\omega) \Gamma(K) \sqrt{\omega^{2}-(K r)^{2}} d \omega d K \\
= & \int_{|K r|}^{\infty} \int_{-\infty}^{\infty}[g(\omega)-g(-\omega)] \Gamma(K) \sqrt{\omega^{2}-(K r)^{2}} d \omega d K .
\end{aligned}
$$

Note that $g(\omega)$ is centered in $-\Omega$ and hence is asymmetric, even for symmetric $\tilde{g}(\tilde{\omega})$ which is often the case. Returning to the original frequencies $\tilde{\omega}$, the last expression becomes

$$
\begin{aligned}
f_{\mathrm{ens}}= & \int_{|K r|}^{\infty} \int_{-\infty}^{\infty}[\tilde{g}(\omega+\Omega)-\tilde{g}(-\omega+\Omega)] \Gamma(K) \\
& \times \sqrt{\omega^{2}-(K r)^{2}} d \omega d K .
\end{aligned}
$$

[1] S. Strogatz, Sync: The Emerging Science of Spontaneous Order (Hyperion, New York, 2003).

[2] Y. Kuramoto, in International Symposium on Mathematical Problems in Theoretical Physics, edited by H. Araki, Lecture Notes in Physics, Vol. 39 (Springer, New York, 1975); Y. Kuramoto, Chemical Oscillations, Waves, and Turbulence (Springer-Verlag, Berlin, 1984).

[3] K. Wiesenfeld, Physica B 222, 315 (1996).

[4] J. H. Sheeba, A. Stefanovska, and P. V. E. McClintock, Biophys. J. 95, 2722 (2008).

[5] S. H. Strogatz, D. M. Abrams, A. McRobie, B. Eckhardt, and E. Ott, Nature (London) 438, 43 (2005).

[6] Y. Kuramoto, Prog. Theor. Phys. 79, 39 (1988).

[7] S. Petkoski and A. Stefanovska, Phys. Rev. E 86, 046212 (2012).

[8] J. A. Acebrón et al., Rev. Mod. Phys. 77, 137 (2005); A. Pikovsky, M. Rosenblum, and J. Kurths, Synchronization A Universal Concept in Nonlinear Sciences (Cambridge University Press, Cambridge, 2001).

[9] H. Hong and S. H. Strogatz, Phys. Rev. Lett. 106, 054102 (2011). [10] J. A. Acebrón and L. L. Bonilla, Physica D 114, 296 (1998).

[11] J. A. Acebrón, L. L. Bonilla, S. De Leo, and R. Spigler, Phys. Rev. E 57, 5287 (1998).
[12] E. Montbrió, J. Kurths, and B. Blasius, Phys. Rev. E 70, 056125 (2004); J. H. Sheeba, V. K. Chandrasekar, A. Stefanovska, and P. V. E. McClintock, ibid. 79, 046210 (2009).

[13] E. A. Martens, E. Barreto, S. H. Strogatz, E. Ott, P. So, and T. M. Antonsen, Phys. Rev. E 79, 026204 (2009).

[14] L. Basnarkov and V. Urumov, Phys. Rev. E 78, 011113 (2008).

[15] H. Sakaguchi and Y. Kuramoto, Prog. Theor. Phys. 76, 576 (1986).

[16] E. Montbrió and D. Pazó, Phys. Rev. Lett. 106, 254101 (2011).

[17] D. Iatsenko, S. Petkoski, P. V. E. McClintock, and A. Stefanovska, Phys. Rev. Lett. 110, 064101 (2013).

[18] M. S. Lama, J. M. López, and H. S. Wio, Europhys. Lett. 72, 851 (2005).

[19] G. H. Paissan and D. H. Zanette, Europhys. Lett. 77, 20001 (2007).

[20] E. Ott and T. M. Antonsen, Chaos 18, 037113 (2008).

[21] D. Pazó and E. Montbrió, Phys. Rev. E 80, 046215 (2009).

[22] A. Pikovsky and M. Rosenblum, Physica D 240, 872 (2011)

[23] H. Wilson and J. Cowan, Biophys. J. 12, 1 (1972).

[24] A. T. Winfree, The Geometry of Biological Time (SpringerVerlag, New York, 1980). 